\title{
Papers
}

\section{Natural experiment examining impact of aggressive screening and treatment on prostate cancer mortality in two fixed cohorts from Seattle area and Connecticut}

\author{
Grace Lu-Yao, Peter C Albertsen, Janet L Stanford, Therese A Stukel, Elizabeth S Walker-Corkery, \\ Michael J Barry
}

\begin{abstract}
Objective To determine whether the more intensive screening and treatment for prostate cancer in the Seattle-Puget Sound area in 1987-90 led to lower mortality from prostate cancer than in Connecticut. Design Natural experiment comparing two fixed cohorts from 1987 to 1997.

Setting Seattle-Puget Sound and Connecticut surveillance, epidemiology, and end results areas. Participants Population based cohorts of male Medicare beneficiaries aged 65-79 drawn from the Seattle ( $\mathrm{n}=94$ 900) and Connecticut $(\mathrm{n}=120$ 621) areas.

Main outcome measures Rates of screening for prostate cancer, treatment with radical prostatectomy and external beam radiotherapy, and prostate cancer specific mortality.

Results The prostate specific antigen testing rate in Seattle was 5.39 (95\% confidence interval 4.76 to 6.11$)$ times that of Connecticut, and the prostate biopsy rate was 2.20 (1.81 to 2.68) times that of Connecticut during 1987-90. The 10 year cumulative incidences of radical prostatectomy and external beam radiotherapy up to 1996 were $2.7 \%$ and $3.9 \%$ for Seattle cohort members compared with $0.5 \%$ and $3.1 \%$ for Connecticut cohort members. The adjusted rate ratio of prostate cancer mortality up to 1997 was 1.03 (0.95 to 1.11$)$ in Seattle compared with

Connecticut.

Conclusion More intensive screening for prostate cancer and treatment with radical prostatectomy and external beam radiotherapy among Medicare beneficiaries in the Seattle area than in the Connecticut area was not associated with lower prostate cancer specific mortality over 11 years of follow up.
\end{abstract}

\section{Introduction}

During the early 1990s the incidence of prostate cancer in the United States rose dramatically after the introduction of testing for prostate specific antigen. The subsequent decline in incidence probably represents depletion of prevalent cases from the pool of men undergoing screening. ${ }^{2}$ In the mid-1990s popu- lation based prostate cancer mortality peaked and then decreased by about $15 \% .^{23}$ Although the decline in mortality may be attributable to screening, several studies have highlighted difficulties in making inferences about causation from cross sectional population based data. ${ }^{4-6}$

The dissemination of prostate cancer screening and treatment was associated with substantial regional variations. ${ }^{7}$ Several studies have documented that the frequency of prostate specific antigen testing, prostate biopsy, and radical prostatectomy among Medicare age men in the Seattle-Puget Sound area was initially higher than in Connecticut. ${ }^{17}$

If the recent national downturn in mortality from prostate cancer is in part attributable to screening and treatment, then an earlier and larger decline in prostate cancer mortality would be expected in Seattle than in Connecticut. The age adjusted prostate cancer death rates in the two regions were almost identical before prostate specific antigen testing (155.8 $v 155.6$ deaths per 100000 person years for white men aged 65-79 and $317.2 v 323.3$ deaths per 100000 person years for African-American men aged 65-79 from 1977 to 1986) (US data by SEER region, age, and race provided L Ries, Cancer Statistics Branch, National Cancer Institute).

This paper presents an 11 year longitudinal study of two cohorts of male Medicare beneficiaries from the Seattle-Puget Sound area and Connecticut exposed to different intensities of prostate cancer screening and treatment during the "early prostate specific antigen era," which we defined a priori as 1987-90. The institutional review boards of Partners Healthcare System and the participating institutions approved the study.

\section{Methods}

Data sources and inclusion criteria

Medicare provides health insurance for people aged 65 and over in the United States and is administered by the Centers for Medicare and Medicaid Services, formerly the Health Care Financing Administration. Medicare pays treatment claims under two programmes: part A covers hospital, nursing home, and home health care, and part B covers physician and outpatient care. The US National Cancer Institute's
Editorial by

Thornton

Papers p 740

Education and debate p 766

HealthStat, PO Box 7501, Princeton, NJ 08543, USA

Grace Lu-Yao director

Division of Urology, Department of Surgery, University of Connecticut Health Center, MC 3955, Farmington, CT 06030, USA

Peter C Albertsen professor of surgery

Division of Public Health Sciences, Programs in Epidemiology and Prostate Cancer Research, Fred

Hutchinson Cancer

Research Center,

1100 Fairview

Avenue North,

MW-814, Seattle,

WA 98109 , USA

Janet L Stanford member and head, Program in Prostate Cancer Research

Center for the Evaluative Clinical Sciences,

Dartmouth Medical School, 7251

Strasenburgh, Hanover, NH

03755 , USA

Therese A Stukel

professor of

biostatistics

Medical Practices Evaluation Center, Massachusetts

General Hospital,

50 Staniford Street,

9th floor, Boston,

MA 02114, USA

Elizabeth S

Walker-Corkery

research coordinator

Michael J Barry

associate professor of

medicine, Harvard

Medical School

Correspondence to: MJ Barry

mbarry@

partners.org

bmj.com 2002;325:740 
surveillance, epidemiology, and end results (SEER) program collects cancer incidence and survival data from 11 population based cancer registries covering approximately $14 \%$ of the US population.

We used four specific data sources for this study: Medicare denominator and vital status files, SEERMedicare linked database, and local SEER registry data. We used the denominator and vital status files, containing information on county of residence, to identify men in the participating SEER areas. Medicare analysts then matched the preliminary study cohort with an existing SEER-Medicare linked file and sent the data to the two local SEER registries. The SEER registries excluded men diagnosed as having prostate cancer before 1987 and supplied updated information on survival and cause of death on the remainder.

To create the SEER-Medicare linked database, each SEER registry sends Medicare information on each person diagnosed as having cancer in its area. Medicare then uses a deterministic matching algorithm to link this data with claims files. To ensure confidentiality, unique identifiers are assigned. We obtained data from the most recent linkage for 1987-96, for which a 93\% match rate was achieved. ${ }^{8}$ The file included prostate specific antigen, prostate biopsy, and radical prostatectomy claims for $100 \%$ of prostate cancer cases up to 1996 and prostate specific antigen and biopsy claims for a 5\% sample of cases without prostate cancer only up to 1993.

We included men who were Medicare beneficiaries aged 65-79 without prostate cancer as of 1 January 1987 and resident within the Seattle-Puget Sound or Connecticut SEER region from 1 January 1987 to 31 December 1990.

Measurement of screening and treatment intensity We used rates of claims for both prostate specific antigen tests and prostate biopsies as measures of screening intensity. Biopsy rates reflect the intensity of screening with digital rectal examinations as well as prostate specific antigen. As prostate specific antigen and biopsy data were available for $100 \%$ of cancer cases and 5\% of non-cancer cases up to 1993 , we calculated the biopsy rate by using a weight of 1 for non-cancer cases and 0.05 for cancer cases. We identified prostate specific antigen tests by current procedural terminology codes 86316 and 84153 (first available in 1988) and biopsies or ultrasound guided biopsies by codes $55700,55705,76942$, and 76943 . We excluded a small percentage of beneficiaries enrolled in health maintenance organisations or without part B coverage from the analyses of screening, as their claims were incomplete. We excluded prostate specific antigen

Table 1 Race and age distributions for the members of the Seattle-Puget Sound and Connecticut study cohorts. Values are numbers (percentages)

\begin{tabular}{lcc} 
Characteristic & Seattle-Puget Sound $(\mathbf{n = 9 4 9 0 0 )}$ & Connecticut $(\mathbf{n}=\mathbf{1 2 0} \mathbf{6 2 1})$ \\
\hline $\begin{array}{l}\text { Race: } \\
\text { White }\end{array}$ & $88863(93.6)$ & $114785(95.2)$ \\
\hline African-American & $1963(2.1)$ & $3882(3.2)$ \\
\hline Other & $4074(4.3)$ & $1954(1.6)$ \\
\hline Age (years) in 1987: & & \\
\hline $65-69$ & $43249(45.6)$ & $53310(44.2)$ \\
\hline $70-74$ & $31544(33.2)$ & $41167(34.1)$ \\
\hline $75-79$ & $20107(21.2)$ & $26144(21.7)$ \\
\hline
\end{tabular}

tests and biopsies that occurred after diagnosis of prostate cancer.

In the prostate specific antigen era screening drives incidence of prostate cancer'; we therefore used prostate cancer incidence as a secondary measure of screening intensity. Prostate cancer incidence data were available up to 1996 .

Data on the use of radical prostatectomy were available for all cancer cases from 1987 to 1996 . We used SEER data alone to document the use of external beam radiation within four months of diagnosis for the same years.

\section{Prostate cancer mortality}

The primary end point for this study was prostate cancer mortality for the two cohorts from 1987 to 1997. We based this on survival data provided directly from the SEER registries.

\section{Statistical methods}

We expressed estimated rates of death, screening, and treatment as numbers of events per 100000 person years. We summed the number of patients alive to the end of the year and half of the patients who died or were lost to follow up during the year to calculate the person years in each cohort annually. We used Poisson regression models to calculate adjusted relative rates of screening, treatment, and death between regions. ${ }^{9}$ This method produces similar relative rate estimates to Cox regression, ${ }^{10}$ as the grouped one year time intervals are short. ${ }^{11}$ We obtained SEER-Medicare linked data on all outcomes grouped by age (65-69, 70-74, 75-79 years), year of study, and registry area. All analyses used the age group-year-area stratum as the unit of analysis, weighted by the number in the stratum. All models controlled for age group, race, area of residence, study year, and an age-race interaction. We censored follow up at year of death or 1997, whichever was earlier. We incorporated variance overdispersion in the estimates of all standard errors to account for possible clustering of outcomes within strata due to common life or practice style influences. ${ }^{9}$ This adjustment increased the width of the confidence intervals negligibly for prostate specific antigen and biopsy, 1.5-fold for radical prostatectomy, and 2.1-fold for cancer incidence. Mortality was not clustered within strata, so we report the Poisson based rate ratios and confidence intervals.

We used life table methods to determine the cumulative incidence of a first prostate specific antigen test (1988-93) and of a prostate cancer diagnosis, radical prostatectomy, and external beam radiation (1987-96) in the two regions. ${ }^{10}$ To show population based prostate cancer mortality trends over time in the two study regions we present cross sectional death rates, adjusted to the age of the study population in 1992. All statistical tests were performed at the $5 \%$ level and were two sided.

\section{Results}

Table 1 shows the race and age distributions for the 215521 eligible men in both cohorts. Cohort members in the Seattle-Puget Sound region were 5.39 (95\% confidence interval 4.76 to 6.11 ) times more likely to undergo prostate specific antigen testing in 1988-90 (table 2) and 2.20 (1.81 to 2.68) times more likely to undergo prostate biopsy in 1987-90 (table 3) 
than their counterparts in Connecticut. Differences in prostate specific antigen testing and biopsy rates narrowed considerably in 1991-3; the Seattle to Connecticut adjusted rate ratio was 1.61 (1.48 to 1.75$)$ for prostate specific antigen and 1.14 (0.93 to 1.40) for biopsy. Figure 1 displays the cumulative incidence of a first prostate specific antigen test among cohort members in the two regions from 1988 to 1993.

Prostate cancer incidence reflects screening rates (fig 2). The adjusted cumulative incidence of prostate cancer was $93 \%$ (81\% to $107 \%)$ higher among men in Seattle-Puget Sound than in Connecticut in 1987-90. The difference in incidence diminished over time; during 1991-6 the cumulative prostate cancer incidence was only $11 \%$ ( $4 \%$ to $18 \%$ ) higher in the Seattle area.

Between 1987 and 1990 men in the Seattle area were more likely to have radical prostatectomy or external beam radiation than their counterparts in Connecticut. Men in the Seattle area had a 5.9-fold (5.0 to 6.9 ) higher rate of radical prostatectomy and

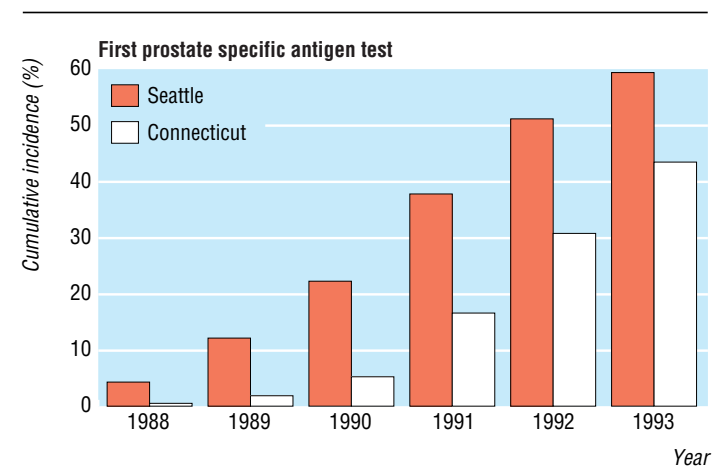

Fig 1 Unadjusted cumulative incidence of a first prostate specific antigen test for study cohort members in the Seattle-Puget Sound and Connecticut areas, 1988-93

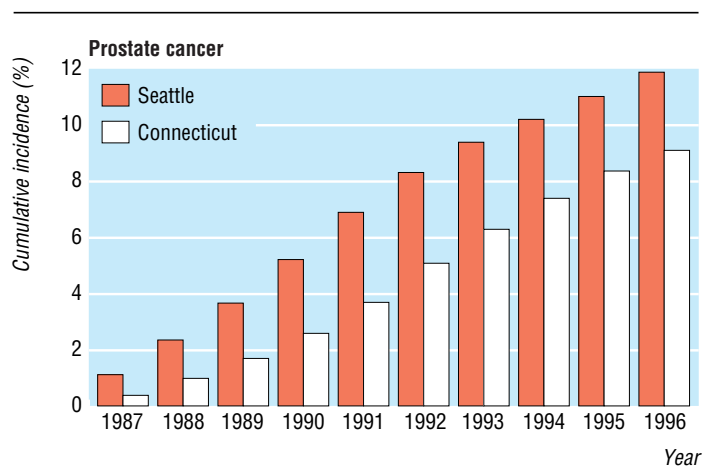

Fig 2 Unadjusted 10 year cumulative incidence of prostate cancer for study cohort members in the Seattle-Puget Sound and Connecticut areas, 1987-96
Table 2 Prostate specific antigen (PSA) testing rates for study cohort members with Medicare part A and B coverage, 1988-90, rate ratio adjusted for age and race

\begin{tabular}{lcclccc} 
& \multicolumn{2}{c}{ Seattle-Puget Sound } & & \multicolumn{2}{c}{ Connecticut } & PSA rate ratio, \\
\cline { 2 - 3 } Age (years) & $\begin{array}{c}\text { No of PSA } \\
\text { tests/person } \\
\text { years }\end{array}$ & $\begin{array}{c}\text { PSA rate per } \\
\mathbf{1 0 0} \mathbf{0 0 0} \\
\text { person years }\end{array}$ & & $\begin{array}{c}\text { No of PSA } \\
\text { tests/ person } \\
\text { years }\end{array}$ & $\begin{array}{c}\text { PSA rate per } \\
\mathbf{1 0 0} \mathbf{0 0 0} \\
\text { person years }\end{array}$ & $\begin{array}{c}\text { Seattle/ } \\
\text { Connecticut } \\
\text { (95\% CI) }\end{array}$ \\
\hline All races: & & & & & & \\
\hline $65-69$ & $505 / 4415$ & 11448 & & $130 / 6357$ & 2043 & \\
\hline $70-74$ & $356 / 3132$ & 11381 & & $113 / 5186$ & 2176 & \\
\hline $75-79$ & $261 / 1959$ & 13322 & & $78 / 3058$ & 2557 & \\
\hline Total & $1122 / 9506$ & 11803 & & $321 / 14601$ & 2199 & 5.39 (4.76 to 6.11) \\
\hline
\end{tabular}

Table 3 Prostate biopsy rates for study cohort members with Medicare part A and B coverage, 1987-90, rate ratio adjusted for age and race

\begin{tabular}{|c|c|c|c|c|c|}
\hline \multirow[b]{2}{*}{$\begin{array}{l}\text { Age } \\
\text { (years) }\end{array}$} & \multicolumn{2}{|c|}{ Seattle-Puget Sound } & \multicolumn{2}{|c|}{ Connecticut } & \multirow[b]{2}{*}{$\begin{array}{c}\text { Biopsy rate ratio } \\
\text { Seattle/Connecticut } \\
(95 \% \mathrm{Cl})\end{array}$} \\
\hline & $\begin{array}{c}\text { No of biopsies/ } \\
\text { person years }\end{array}$ & $\begin{array}{c}\text { Biopsy rate per } \\
100000 \\
\text { person years }\end{array}$ & $\begin{array}{c}\text { No of biopsies/ } \\
\text { person years }\end{array}$ & $\begin{array}{c}\text { Biopsy rate per } \\
100000 \\
\text { person years }\end{array}$ & \\
\hline $65-69$ & $110 / 6019$ & 1822 & $71 / 8651$ & 818 & \\
\hline $70-74$ & $92 / 4327$ & 2124 & $60 / 7136$ & 842 & \\
\hline $75-79$ & $40 / 2747$ & 1471 & $38 / 4275$ & 882 & \\
\hline Total & $242 / 13093$ & 1848 & $169 / 20062$ & 840 & 2.20 (1.81 to 2.68$)$ \\
\hline
\end{tabular}

2.3-fold (2.2 to 2.5) higher rate of external beam radiation during 1987-90. The 10 year cumulative incidences of radical prostatectomy and external beam radiation from 1987 to 1996 were $2.7 \%$ and $3.9 \%$ for Seattle-Puget Sound cohort members and $0.5 \%$ and $3.1 \%$ for Connecticut cohort members (fig 3).

No significant difference in prostate cancer mortality existed between the Seattle and Connecticut cohorts over the entire 11 year follow up period. The adjusted rate ratio of prostate cancer mortality to 1997 was 1.03 (0.95 to 1.11) in Seattle compared with Connecticut (table 4). As any effect of more aggressive screening and treatment on mortality may have been delayed, we compared prostate cancer mortality separately for the earlier (1987-92) and later (1993-7) years of follow up. No significant differences occurred in either period-rate ratios 0.97 (0.81 to 1.16$)$ versus 1.08 (0.98 to 1.20$)$. Fig 4 displays cross sectional prostate cancer mortality for men aged 65-79 from 1987 to 1997 , documenting similar decreases in both areas.

\section{Discussion}

Given the greater intensity of screening, diagnosis, and treatment documented among cohort members in the Seattle-Puget Sound area than in Connecticut, we would have expected to see lower prostate cancer mortality in the Seattle region over time. However, we found no significant difference in prostate cancer mortality over 11 years of follow up. The confidence interval around the rate ratio for mortality was inconsistent with more than a $5 \%$ reduction in prostate cancer

Table 4 Prostate cancer specific mortality by age, and age adjusted mortality rate ratios, 1987-97

\begin{tabular}{|c|c|c|c|c|c|}
\hline \multirow[b]{2}{*}{ Age (years) } & \multicolumn{2}{|c|}{ Seattle-Puget Sound } & \multicolumn{2}{|c|}{ Connecticut } & \multirow{2}{*}{$\begin{array}{l}\text { Age adjusted mortality rate } \\
\text { ratio, Seattle/Connecticut } \\
(95 \% \mathrm{CI})\end{array}$} \\
\hline & No of deaths/ person years & $\begin{array}{l}\text { Mortality per } 100000 \\
\text { person years }\end{array}$ & $\begin{array}{c}\text { No of deaths/ person } \\
\text { years }\end{array}$ & $\begin{array}{c}\text { Mortality per } 100000 \\
\text { person years }\end{array}$ & \\
\hline $65-69$ & $379 / 396489$ & 95.6 & $442 / 481372$ & 91.8 & \\
\hline $70-74$ & $394 / 261599$ & 150.6 & $515 / 336783$ & 152.9 & \\
\hline $75-79$ & $307 / 144726$ & 212.1 & $386 / 181462$ & 209.6 & \\
\hline Total & $1080 / 802813$ & 134.5 & $1343 / 1002316$ & 134.0 & $1.03(0.95$ to 1.11$)$ \\
\hline
\end{tabular}



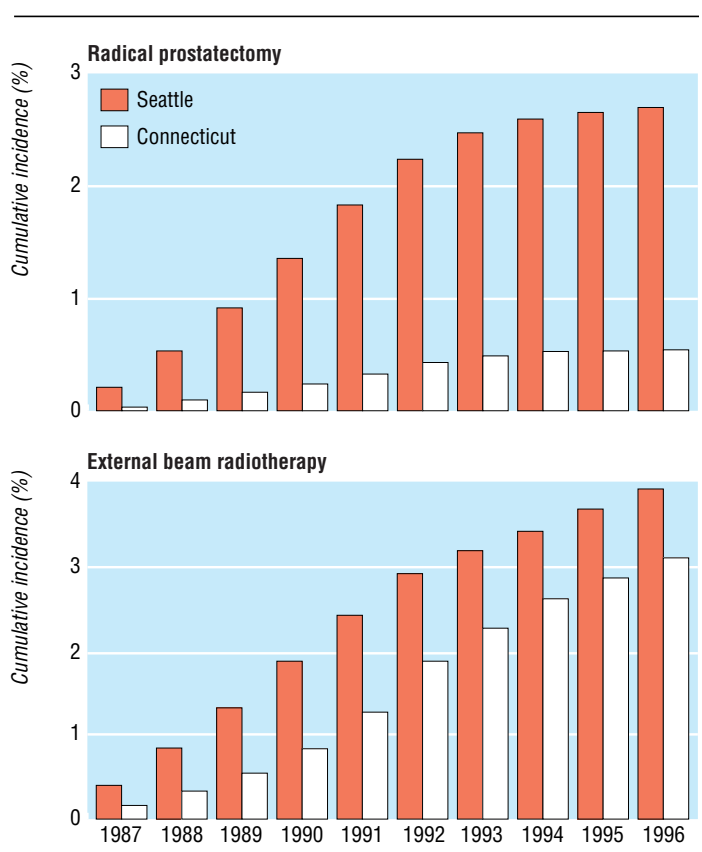

Fig 3 Unadjusted 10 year cumulative incidence of radical prostatectomy (top) and external beam radiotherapy (bottom) for cohort members in the Seattle-Puget Sound and Connecticut areas, 1987-96

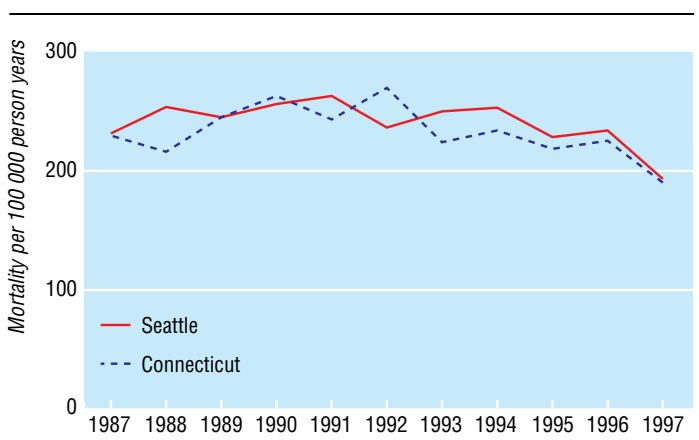

Fig 4 Age adjusted prostate cancer mortality per 100000 person years for men in Seattle-Puget Sound and Connecticut on the basis of cross sectional data, 1987-97 (adjusted to the age composition of the entire study cohort: $48.3 \%$ aged $70-74,33.3 \%$ aged $75-79$, $18.4 \%$ aged $80-84$ as of 1 January 1992). (US data by SEER region, age, and race provided by L Ries, Cancer Statistics Branch, National Cancer Institute)

mortality in Seattle. Moreover, the overall drop in prostate cancer mortality seemed similar in the two regions (fig 4). The results of this study therefore do not support the hypothesis that the intensity of screening and treatment with surgery or radiation was related to the reduction in prostate cancer mortality seen in the two regions.

A recent report has documented decreases in population based prostate cancer mortality in Tyrol compared with other states in Austria after the introduction of mass prostate specific antigen screening in Tyrol. ${ }^{12}$ Our results are in conflict with these for unclear reasons; although the intensity of screening in Tyrol seems similar to that in the Seattle area (about two thirds of men screened at least once over five years), the intensity of treatment in Tyrol and the intensities of both screening and treatment in the rest of Austria were not presented in that report.

\section{Limitations of the study}

Our study has several limitations. Firstly, the cohorts included only men aged 65 and over in 1987. Screening and treatment for prostate cancer may have a larger impact on younger men. However, as prostate cancer death is rare before age $70,{ }^{3}$ if recent decreases in prostate cancer mortality in the United States are attributable to screening and treatment with surgery and radiation this impact would almost certainly be seen among Medicare age men.

Secondly, during 1987-90 Medicare did not routinely pay for prostate specific antigen screening tests. Claims may not have always been submitted, therefore, and numbers of prostate specific antigen tests may be underestimated. Nevertheless, the relative rates of testing between the two regions should not be biased, and the differences in biopsies and incidence confirm the higher intensity of screening in the Seattle area.

Thirdly, follow up over 11 years may not have been long enough to see a difference in prostate cancer mortality. However, if the decreases in population based prostate cancer mortality noted in both these areas (fig 4) are indeed attributable to screening and treatment with surgery or radiation, the time frame should have been sufficient to see an effect. Nevertheless, further follow up is planned.

Fourthly, we used SEER data to capture new diagnoses of prostate cancer, so records of diagnosis or cause of death for cohort members who moved out of their SEER areas after 1990 were unavailable. A differential rate of death from prostate cancer among men who moved after 1990 could introduce bias. However, Medicare enrolment files indicated that less than $1 \%$ of cohort members without prostate cancer moved out of their regions before dying. As only about 3\% of these men would be expected to die of prostate cancer, the impact of any differential rate of death from prostate cancer should be minimal.

Fifthly, as the primary outcome measure was prostate cancer specific mortality a differential assignment of cause of death among prostate cancer patients in the two regions could bias comparisons. Earlier studies have shown a relatively high agreement (over 90\%) between medical records and underlying causes of death as recorded on death certificates. ${ }^{13}$ Our group has shown a high level of agreement between medical records and the cause of death as listed on the death certificate among patients with prostate cancer in Connecticut $-91 \%$ for men dying in 1995 and 82\% for men dying in 1985-with no change in the coding of cause of death among prostate cancer patients dying in the pre-prostate specific antigen (1985) and prostate specific antigen (1995) eras. ${ }^{14}$ Moreover, as part of the current study, we replicated this analysis for patients with prostate cancer who died in the Seattle area in 1995; once again, the agreement between death certificates and medical records on cause of death was greater than $90 \% .^{15}$

Sixthly, prostate specific antigen and biopsy data were incomplete for men enrolled in a Medicare health maintenance organisation in the two SEER areas dur- 


\section{What is already known on this topic}

Screening for prostate cancer with the prostate specific antigen test is widely practised in the United States and has resulted in a remarkable increase in incidence of diagnosed disease

Randomised trials are under way to determine whether early detection and aggressive treatment reduce mortality from prostate cancer

Recent reductions in prostate cancer mortality in the United States have been attributed to screening and treatment, raising questions about whether continuing the trials is ethical

\section{What this study adis}

Prostate cancer screening and treatment were much more intensive among men in the Seattle-Puget Sound area than in Connecticut early in the "prostate specific antigen era"

Over 11 years of follow up, no difference in prostate cancer mortality was seen in the two cohorts

The lack of association between more intensive screening and treatment and lower prostate cancer mortality suggests that trials should continue in order to settle this question

ing the early prostate specific antigen era. The men enrolled in health maintenance organisations were included in the primary analyses of treatment and mortality rates. Perhaps men enrolled in health maintenance organisations were not as intensively screened in the Seattle area, which would tend to dilute any mortality benefit attributable to screening. However, excluding men enrolled in health maintenance organisations from the mortality analysis did not affect the results: rate ratio=1.0 (0.9 to 1.1 ).

Finally, men in the two cohorts may have differed on important risk factors for prostate cancer. Although only randomised trials can yield truly unbiased estimates of the efficacy of screening and treatment on prostate cancer mortality, their external validity might be limited. ${ }^{16}$ This longitudinal cohort study, which applied uniform selection criteria at "zero time" for determining eligibility and included a broad representation of the population at risk, may provide complementary and important insights into what might be expected at the population level.

\section{Why has mortality decreased?}

This study does not shed light on the reasons for the decline in prostate cancer mortality in these two regions. More effective treatment for advanced disease may have improved mortality in both areas; rates of androgen deprivation, a primary treatment for advanced prostate cancer, could not be tracked with the data available. Additionally, changes in lifestyle or environmental factors that may affect the progression of prostate cancer may have had a role in these mortality trends.

\section{Conclusion}

More intensive screening for prostate cancer and treatment with surgery or radiation among a cohort of Medicare beneficiaries in the Seattle-Puget Sound area compared with a cohort in Connecticut has not led to significantly lower mortality from prostate cancer over 11 years. Longer follow up will be necessary to fully assess the outcome of this natural experiment. Meanwhile, ongoing randomised trials assessing the effectiveness of screening and treatment for prostate cancer should be supported.

The study used the linked SEER-Medicare database; the interpretation and reporting of these data are the sole responsibility of the authors. We acknowledge the efforts of the Applied Research Program, National Cancer Institute; the Office of Information Services and the Office of Strategic Planning, HCFA; Information Management Services (IMS); and the Surveillance, Epidemiology, and End Results (SEER) Program tumour registries in the creation of the SEER-Medicare database. Additionally, we thank E Robert Greenberg of the Norris Cotton Cancer Center at Dartmouth-Hitchcock Medical Center for providing insightful comments, Maribel Franey and Edward Franey at HCFA/CMS for processing our data request and matching our cohort with the SEER-Medicare linked file, and the Seattle-Puget Sound and Connecticut SEER registries for updating and evaluating cancer incidence and mortality in the study cohorts.

Contributors: GL-Y, PCA, JLS, ESW-C, and MJB participated in the conception and design of the study and acquisition of data GL-Y, PCA, JLS, TAS, and MJB participated in planning the analysis and interpreting the results. GL-Y performed the statistical analyses. All authors contributed to writing and revising the manuscript. GL-Y is the guarantor.

Funding: Agency for Health Care Research and Quality, grant Nos HS 08397 and HS 10278.

Competing interests: None declared.

1 Potosky A, Miller B, Albertsen P, Kramer B. The role of increasing detection in the rising incidence of prostate cancer. JAMA 1995;273:548-52.

2 Ries L, Eisner M, Kosary C, Hankey B, Miller BA, Clegg L et al. SEER cancer statistics review, 1973-1997. Bethesda, MD: National Cancer Institute, 2000.

3 Tarone R, Chu K, Brawley O. Implications of stage-specific survival rates in assessing recent declines in prostate cancer mortality rates. Epidemiology 2000;11:167-70.

4 Hankey B, Feuer E, Clegg L, Hayes RB, Legler JM, Prorok PC, et al Cancer surveillance series: interpreting trends in prostate cancer-part I: evidence of the effects of screening in recent prostate cancer incidence, evidence of the effects of screening in recent prostate cancer
mortality, and survival rates. J Natl Cancer Inst 1999;91:1017-24

5 Feuer E, Merrill R, Hankey B. Cancer surveillance series: interpreting trends in prostate cancer-part II: cause of death misclassification and the recent rise and fall in prostate cancer mortality. J Natl Cancer Inst 1999;91:1025-32.

6 Etzioni R, Legler J, Feuer E, Merrill R, Cronin K, Hankey B. Cancer surveillance series: interpreting trends in prostate cancer-part III: quantifying the link between population prostate-specific antigen testing and recent declines in prostate cancer mortality. J Natl Cancer Inst 1999;91:1033-39.

7 Lu-Yao GL, McLerran D, Wasson J, Wennberg J, the Prostate Patient Outcomes Research Team. An assessment of radical prostatectomy: time trends, geographic variation, and outcomes. JAMA 1993;269:2633-36.

8 National Cancer Institute Applied Research Program. wwwdccps.ims.nci.nih.gov/ARP/SEERMed/seermedicare.html (2000).

9 McCullagh P, Nelder J. Generalized linear models, 2nd ed. London: Chapman and Hall, 1989.

10 Cox DR. Regression models and life-tables. J R Stat Soc Ser B Methodological 1972;34:187-220.

11 D'Agostino R, Lee M, Belanger A. Relation of pooled logistic regression to time dependent Cox regression analysis: the Framingham Heart Study. Stat Med 1990;9:1501-15.

12 Bartsch G, Horninger W, Klocker H, Reissigl A, Oberaigner W, Schonitze D, et al. Prostate cancer mortality after introduction of prostate-specific antigen mass screening in the federal state of Tyrol, Austria. Urology 2001;58:417-24.

13 Percy C, Stanek E, Gloeckler L. Accuracy of cancer death certificates and its effect on cancer mortality statistics. Am J Pub Health 1981;71:242-50.

14 Albertsen P, Walters S, Hanley J. A comparison of cause of death determination in men previously diagnosed with prostate cancer who died in 1985 or 1995. J Urol 2000;163:519-23.

15 Penson D, Albertsen P, Nelson P, Barry M, Stanford J. Determining cause of death in prostate cancer: are death certificates valid? J Natl Cancer Inst 2001;93:1822-3. 
Papers

16 Concato J, Shah N, Horwitz RI. Randomized, controlled trials, observational studies, and the hierarchy of research designs. $N$ Engl J Med

(Accepted 24 June 2002)

2000;342:1887-92.

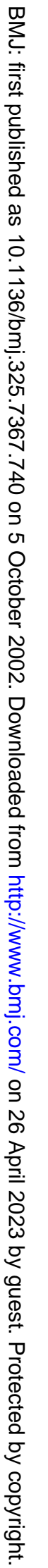

